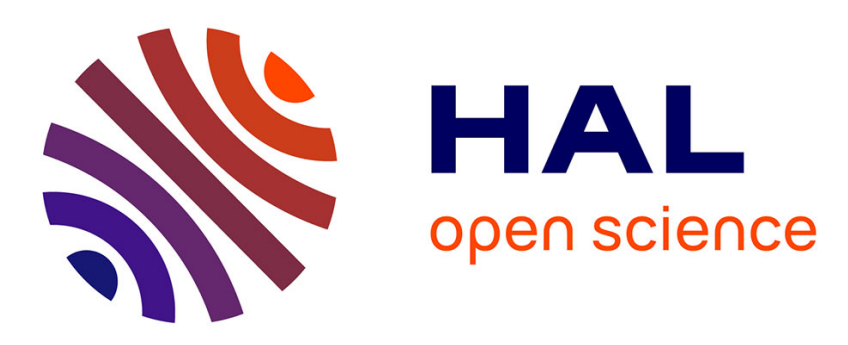

\title{
Scale separation between grain detachment and grain transport in granular media subjected to an internal flow.
}

\author{
A. Wautier, Stéphane Bonelli, François Nicot
}

\section{To cite this version:}

A. Wautier, Stéphane Bonelli, François Nicot. Scale separation between grain detachment and grain transport in granular media subjected to an internal flow.. Granular Matter, 2017, 19 (2), 15 p. 10.1007/s10035-017-0706-9 . hal-01720370

\section{HAL Id: hal-01720370 \\ https://hal.science/hal-01720370}

Submitted on 1 Mar 2018

HAL is a multi-disciplinary open access archive for the deposit and dissemination of scientific research documents, whether they are published or not. The documents may come from teaching and research institutions in France or abroad, or from public or private research centers.
L'archive ouverte pluridisciplinaire HAL, est destinée au dépôt et à la diffusion de documents scientifiques de niveau recherche, publiés ou non, émanant des établissements d'enseignement et de recherche français ou étrangers, des laboratoires publics ou privés. 


\title{
Scale separation between grain detachment and grain transport in granular media subjected to an internal flow.
}

\author{
Antoine Wautier · Stéphane Bonelli · François Nicot
}

Received: 15 September 2016 / First Online: 07 March 2017

The final publication is available at Springer via http://dx.doi.org/10.1007/s10035-017-0706-9

A read-only version can be found at http://rdcu.be/pSuR

\begin{abstract}
Based on a discrete element method (DEM), this paper investigates the basic mechanisms and the associated scales related to grain detachment and grain transport processes at stake in widely graded poly-disperse assemblies of spheres subjected to internal fluid flows. From the identification of force chains, particles sensitive to grain detachment are identified. Based on the computation of autocorrelation lengths, a typical length scale associated with this phenomenon is then defined. From the characterization of the void space as a pore network, particles eligible for grain transport are identified among the detachable particles. Based on the definition of a mean travel distance, the typical length scale associated with grain transport is finally characterized. The comparison between the two length scales highlights a scale separation between grain detachment and grain transport.
\end{abstract}

Keywords Mesoscales · Micromechanics - DEM - Force chains · Autocorrelation · Pore Network

\section{Introduction}

In various circumstances, granular materials are submitted to internal flows which may modify their microstructure and

\footnotetext{
A. Wautier

AgroParisTech-ENGREF, 19 avenue du Maine, 75732 Paris, France.

E-mail: antoine.wautier@irstea.fr

A. Wautier · F. Nicot

Université Grenoble Alpes, Irstea, UR ETGR, 2 rue de la Papeterie-BP 76, F-38402 St-Martin-d'Hères, France.

A. Wautier - S. Bonelli

Irstea UR RECOVER, 3275 Rte Cézanne, CS 40061, 13182 Aix-en-

Provence Cedex 5, France.
}

by consequence the overall hydraulic and mechanical properties of the granular assembly. At the microscale, this process consists in a rearrangement of particles driven by three elementary mechanisms, namely the detachment of grains from the granular skeleton, their transport through the pore network and possibly their reattachment to the granular skeleton farther away. At a larger scale, this process results in the selective erosion of the smallest particles of a granular material and is referred to as suffusion within the geomechanics community (Bonelli, 2012, Sibille et al., 2015). Being able to predict the occurrence of such a phenomenon is of crucial interest in many domains as it can trigger off critical mechanical instabilities or generate undesired solid transport.

Since the end of the $20^{\text {th }}$ century, the ability of an internal fluid flow to modify the microstructure of a granular material was systematically linked to its particle size distribution (PSD) (Kenney and Lau, 1985, Kézdi, 2013, Terzaghi, 1939, To and Scheuermann, 2014). More recently, with the ability of discrete element methods (DEMs) (Cundall and Strack. 1979) to handle a substantial number of particles, these susceptibility criteria were considered from a micromechanical point of view (Fonseca et al., 2014, Langroudi et al. 2015. Shire and O'Sullivan, 2013) and linked to the average number of contacts per particle, contact distributions and the probability of a particle participating in stress transmission.

Complementary to these particle-based approaches, constriction size distribution (CSD) curves were defined to account for the transport properties of granular materials (Li et al. 2014, Sjah and Vincens, 2013, To and Scheuermann, 2014. Vincens et al. 2015). These approaches describe the void space in terms of accessible volumes for particles of different sizes. Many have attempted to relate the CSD to the PSD, to assess the possibility of some particles being transported (OSullivan et al., 2015, To and Scheuermann, 2014. 
To et al. 2015b). Recently, To et al. (2015a) even proposed a probability criterion based on these approaches.

Although the first approaches tend to reduce the internal flow impact to grain detachment, the second ones tend to reduce it to grain transport. In fact, global approaches that can integrate and merge these two aspects are lacking. In the last 10 years, several numerical schemes have been developed to take into account fluid/grain interactions and enable full three-dimensional micro-modeling of the detachment, the transport and the reattachment of grains in a granular assembly (LBM, SPH and PFV methods, for instance). This paper investigates the possibility of providing a quantitative definition of the length scales associated with the two first cited mechanisms from a micromechanical point of view as there is a need to correctly define a minimum sample volume that should be used at the material scale in order to formulate homogenized law for suffusion.

Since detachable particles are characterized by weak contact forces, grain detachment processes are closely related to the spatial distribution of forces inside a granular assembly. Based on the identification chained particles (Peters et al. 2005, Tordesillas et al., 2010) combined with the computation of autocorrelation functions (Kanit et al., 2003, Lantuejoul, 1991, Matheron, 1967), the statistical properties of the population of particles transmitting the mechanical loading can be explored. The typical size of the loose grain structures is characterized with the dual analysis of autocorrelation lengths associated with force transmission within the integral range theory (Matheron, 1967).

Inside a saturated granular media in which electrostatic forces can be neglected, grain transport is mainly governed by the geometrical properties of the porous space and in particular the spatial distribution of the pore space accessible to free particles. In the case of a sphere assembly, the porous phase can be modeled as a graph characterized by pores and constrictions of different radii. In the wake of the previous work of Reboul et al. (2008) and Vincens et al. (2015), the statistical properties of this network are related to the possibility of particles being transported through the granular matrix. Given a macroscopic pressure gradient, typical transport distances are defined as a function of these virtual particle radii.

This paper is organized as follows. In section 2, idealized virtual samples defined as poly-dispersed assemblies of spheres of varying void indices are generated and subjected to a drained triaxial test. Based on the geometrical and micromechanical description of the generated samples under testing, two mesoscales (associated with force transmission and particle transport) are defined and quantified in sections 3 and 4 respectively.

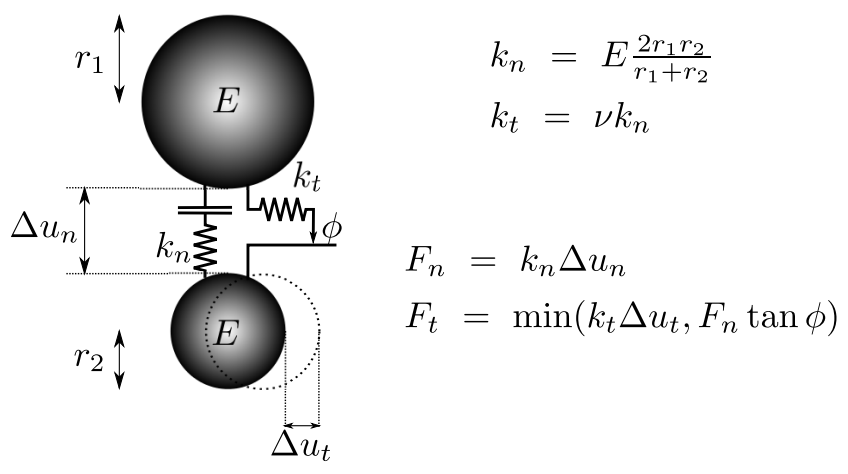

Fig. 1 Elasto-frictional contact law used in DEM simulations.

Table 1 Mechanical parameters used in the elasto-frictional contact law implemented in YADE.

\begin{tabular}{ll}
\hline Parameters & Value \\
\hline Density & $3,000 \mathrm{~kg} \cdot \mathrm{m}^{-3}$ \\
Young Modulus $(E)$ & $356 \mathrm{MPa}$ \\
Stiffness ratio $(v)$ & 0.42 \\
Inter-particle friction angle $(\phi)$ & $35^{\circ}$ \\
Particle-wall friction angle & $0^{\circ}$ \\
Number of particles & 10,000 \\
\hline
\end{tabular}

\section{Sample preparation and triaxial testing}

\subsection{Numerical modeling}

The micromechanical analysis performed in this paper considers non cohesive granular materials modeled as poly-disperse assemblies of spheres. The interaction between two particles is modeled by the classical elasto-frictional contact law proposed by Cundall and Strack (1979) and is illustrated in Figure 1 .

Two spherical particles are said to be in contact if they overlap. Based on the direction of the vector joining the particle centers, a normal force $F_{n}$ is defined as proportional to the overlapping distance $\Delta u_{n}$ between the two particles where the contact normal stiffness $k_{n}$ is proportional to the material's Young modulus $E$ and to the harmonic average of the two particles radii $r_{1}$ and $r_{2}$ (see Fig. 1). In addition to the normal force, a tangential force $F_{t}$ is introduced. This tangential force is proportional to the relative tangential displacement $\Delta u_{t}$ between the two particles where the horizontal contact stiffness is a fraction $v$ of its normal counterpart (see Fig. 1). Contrary to $\Delta u_{n}$, the definition of $\Delta u_{t}$ is not straightforward. It is defined in an incremental form as the relative tangential displacement of the contact point between a reference configuration (when the contact is first established) and the current configuration. The last parameter of the implemented contact law is the internal friction angle $\phi$, which defines the largest accessible ratio $F_{t} / F_{n}$ according to the Mohr Coulomb theory (see Fig. 11. The input parameters used in this elasto-frictional contact law are reported in Table 1. They are chosen equal to those of Hadda (2006). 


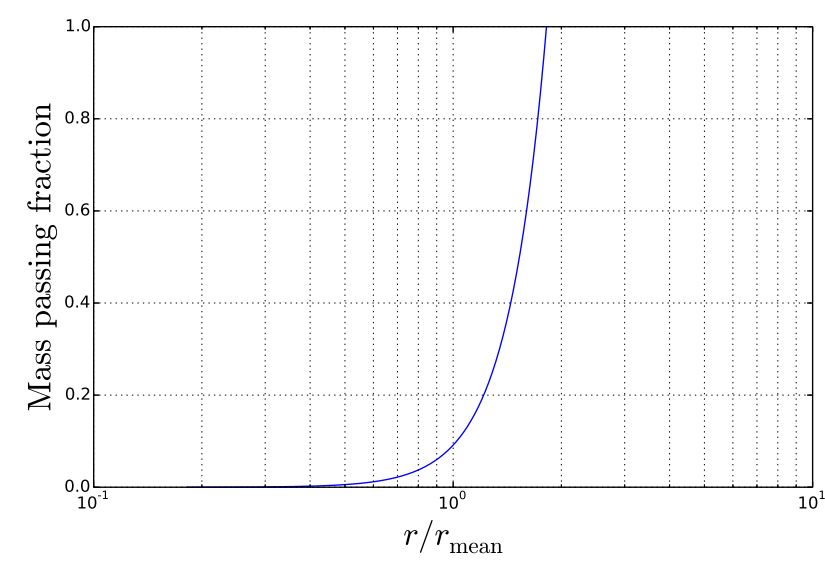

Fig. 2 Normalised PSD curve by mass corresponding to the generated sample. Particle radii are normalised by the mean radius value.

After computing all inter-particle contact forces, the induced particles displacements are integrated based on Newton's second law of motion using the DEM open source code YADE (Šmilauer et al. 2015).

\subsection{Sample generation}

Cubic assemblies of spheres are generated randomly with a uniform radius distribution between $r_{\min }$ and $r_{\max }=10 r_{\min }$. This particle size distribution is taken such that the smallest particles have a good geometrical chance of being transported through the voids created by the largest ones according to the Therzaghi filter rule: $r_{\min } \ll 4 r_{\max }$ (Terzaghi, 1939). The normalized PSD curve by mass is shown in Figure 2. The resulting coefficient of uniformity is $c_{u}=1.3$.

After generating a cloud of 10,000 non-overlapping spheres surrounded by six bounding planes defining a cube, the particles are inflated and allowed to rearrange according to the radius expansion technique. This process is stopped when the confining pressure applied on the bounding planes reaches $20 \mathrm{kPa}$. From this point, the internal friction angle is artificially decreased by small steps, making it easier for particles to rearrange. Since the particle radii should be increased to keep the confining pressure constant, this procedure results in a densification process. Three geometrical configurations are generated during this process when the void ratio of the sample reaches the targeted values of $0.8,0.7$ and 0.6 . These values were selected such that the resulting three samples exhibit macroscopic behaviors typical of loose, intermediate, and dense sands respectively. Accordingly, they are referred to as "loose sample", "medium sample" and "dense sample" hereafter. Associated with the cubic grain assemblies thus generated, a Cartesian coordinate system $\left(\boldsymbol{e}_{x}, \boldsymbol{e}_{y}, \boldsymbol{e}_{z}\right)$ is defined such that the axis directions coincide with the edges of the cube. This definition is recalled in Figure 4.

\subsection{Drained triaxial test}

After the sample preparation phase, the internal friction angle is restored to its initial value of $35^{\circ}$. The same dry drained triaxial test is applied to the three prepared samples. This test consists in two steps:

- First an isotropic confining pressure of $\sigma_{0}=100 \mathrm{kPa}$ is applied by allowing the bounding walls to move;

- Then a vertical compression strain rate $\dot{\varepsilon}_{z z}=-0.01 \mathrm{~s}^{-1}$ is applied up to $20 \%$ of deformation while keeping a constant lateral confining pressure of $\sigma_{0}$; this strain rate is chosen such that the loading can be considered as quasi-static (Hadda, 2006).

The classical deviatoric stress and volumetric strain responses are shown for the three samples in Figure 3. From the resulting forces applied on the bounding walls a macroscopic stress tensor $\boldsymbol{\sigma}$ is computed. The resulting deviatoric stress $q$ is then defined as

$$
\left\{\begin{aligned}
q & =\sqrt{\frac{3}{2} \boldsymbol{\sigma}_{\mathrm{dev}}: \boldsymbol{\sigma}_{\mathrm{dev}}} \\
\boldsymbol{\sigma}_{\mathrm{dev}} & =\boldsymbol{\sigma}-\frac{1}{3} \operatorname{Tr}(\boldsymbol{\sigma}) \mathbf{1}
\end{aligned}\right.
$$

where 1 stands for the identity tensor and : stands for the double dot contraction product.

Likewise, the macroscopic homogeneous strain tensor $\boldsymbol{\varepsilon}$ is defined from the bounding walls displacements. The volumetric strain is thus simply defined as

$\varepsilon_{v}=\operatorname{Tr}(\boldsymbol{\varepsilon})$.

In Figure 3, the dense sample is characterized by a dilative behavior after a brief initial contracting phase. The stress response reaches a peak followed by a softening phase. The loose sample is characterized by a contractive behavior and the absence of a stress peak. The medium sample evolves in an isochoric way with a stress peak immediately followed by a plateau. These three mechanical responses are consistent with those classically observed both in the laboratory (Terzaghi et al., 1996) and from discrete numerical simulations (Scholtès et al., 2010, Wang and Li, 2015). Moreover, one can highlight the existence of a unique critical state, as described in the classical critical state theory in soil mechanics (Schofield and Wroth, 1968).

\section{Mesoscale investigation with respect to force transmission}

The microscale analysis of the force network in granular assemblies is underpinned by two governing ideas with respect to grain detachment. The first idea is that the most 

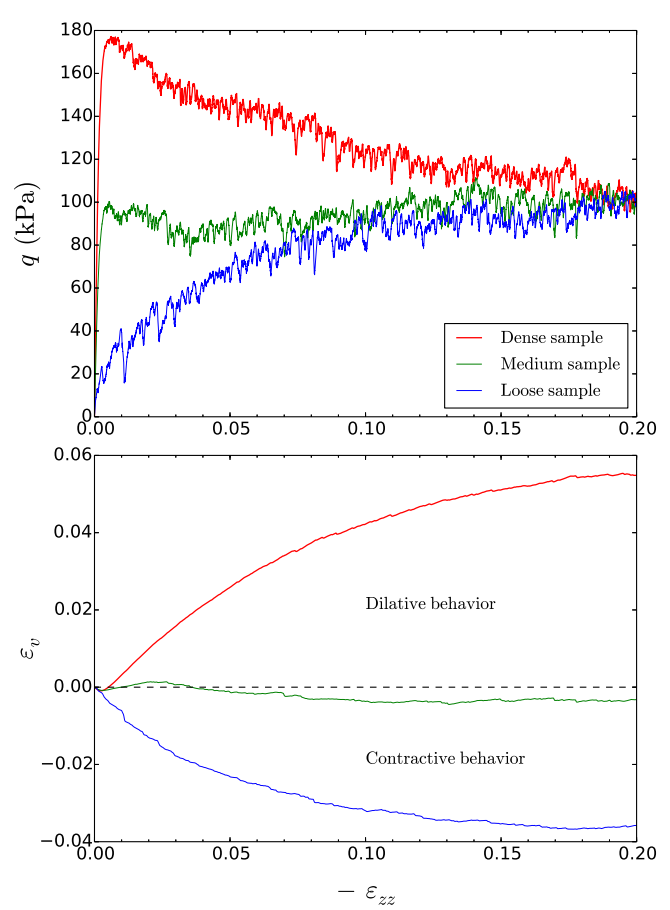

Fig. 3 Stress (top) and volumetric (bottom) responses of the three prepared samples during a triaxial test with confining pressure of $100 \mathrm{kPa}$ and vertical strain $\left|\varepsilon_{z z}\right| \in[0,0.2]$

detachable grains do not transmit important forces. The second idea is that the typical length scale associated with the detachment process should be linked with the distance between the grains transmitting the stresses through the granular assembly. As a result, the grains of the three samples presented in the previous section are first divided into two groups based on the force chain definition introduced by $\mathrm{Pe}$ ters et al. (2005). Then the spatial distribution of force chains is investigated using autocorrelation functions (Kanit et al. 2003. Matheron, 1967).

\subsection{Statistical properties of force chains}

\subsubsection{Force chain definition}

The detailed algorithm used to identify the chained particles of a sample can be found in Peters et al. (2005). We simply recall here the definition of a force chain which is based on the following three characteristics (Peters et al., 2005):

- The particles belonging to a force chain have a higher principal stress than the mean particle principal stress;

- The principal stress direction of chained particles is aligned with the geometrical contact direction (less than $45^{\circ}$ deviation);

- A force chain contains at least three contacting particles.

Based on this definition, force chains can be identified in the three samples for several strain values. It should be noticed

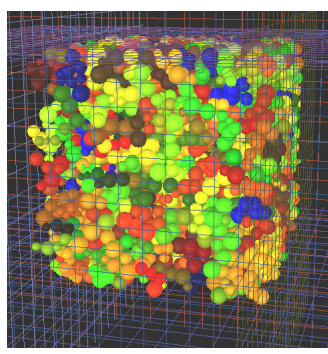

(a) $\varepsilon_{z z}=0 \%$

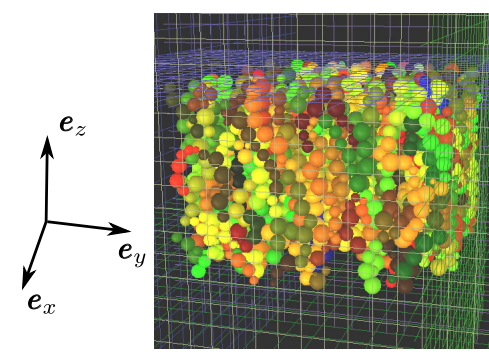

(b) $\varepsilon_{z z}=20 \%$
Fig. 4 Force chains visualisation for the dense sample under an isotropic confining pressure of $100 \mathrm{kPa}$ (a) and at the end of the triaxial test (b).

here that the definition used is particle centered and not contact centered as classically done for the definition of strong and weak force networks (Cambou et al., 2013). Indeed, the identification of force chains relies on the computation of the principal compression intensity deduced from the mean cauchy stress in each single grain. A typical visualization of the force chains in the dense sample can be found in Figure 4 . At the beginning of the triaxial test, the force chains are distributed in an isotropic way which is consistent with the fact that no principal direction of loading exist. Once the deviatoric loading is applied, the force chains tend to align in the vertical direction of the macroscopic principal stress which is consistent with the previous results obtained in $2 \mathrm{D}$ with the combined use of fabric tensors and contact forces network by Radjai et al. (1998).

\subsubsection{Statistical description of force chains during triaxial loading}

In addition to these qualitative observations, some of the force chains statistical properties can be analyzed. In Figure 5 the percentage of chained particles, the total number of force chains and the mean length of a force chain are plotted for the different samples during the triaxial test presented in the previous section. On these graphs, the stress-strain curves illustrated in Figure 3 are recalled in dotted lines. As pointed out in Peters et al. (2005), only a small fraction of the total number of particles is involved in force chains. At the beginning of the triaxial test, the fraction of chained particles ranges from 23 to $31 \%$ depending on the sample density (Fig. 5 (a)). The higher the density, the more particles are involved in force chains. This should be related to the fact that for a dense sample the high number of contacts between particles enables a homogeneous distribution of the stress inside the sample. Conversely, a loose sample presents a more limited number of contacts, which results in the concentration of the stress onto a more limited number of particles. As the deviatoric loading starts, force chains begin to disappear, as illustrated in Figure 5 (a) and (b) for the dense and medium samples, respectively. After merely $10 \%$ 


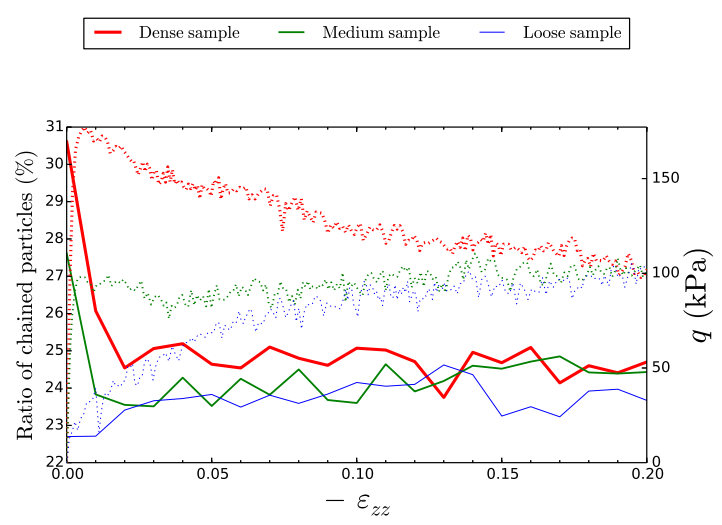

(a)

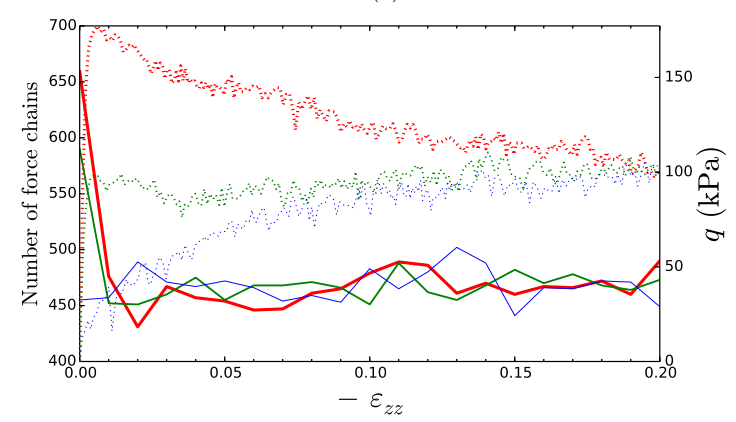

(b)

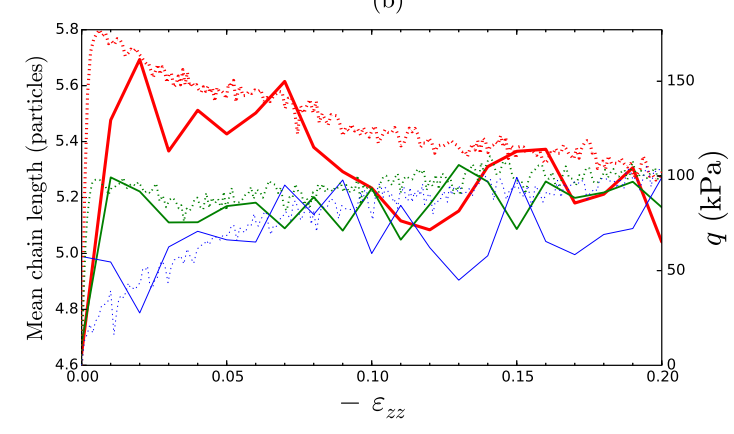

(c)

Fig. 5 Strain evolution of the ratio of chained particles (a), the number of force chains (b) and the mean chain length (c) for the three samples considered during the triaxial test. The macroscopic stress strain response is recalled in dots on the three graphs.

of strain, a critical state is reached with only $24-25 \%$ of particles involved in force chains.

The evolution of the mean length of a force chain in terms of the number of particles involved gives information on the ability of a material to transmit an additional load. Indeed, granular assemblies containing long force chains are able to sustain high stress levels because most of the stiffness will result from the normal contact stiffness $k_{n}$ (see Fig. 1). In contrast, for granular assemblies containing short force chains the overall stiffness is more influenced by the tangential contact stiffness $k_{t} \leq k_{n}$ and the friction angle $\phi$ (see

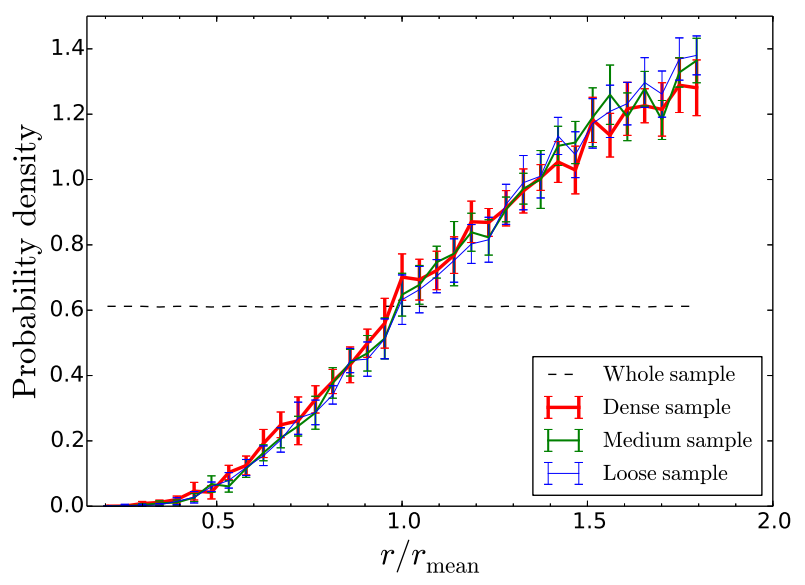

Fig. 6 Mean probability densities for a grain of a given radius to be part of a force chain belonging to each of the three samples. Standard deviations are shown thanks to error bars. The dashed lines correspond to the probability density for a grain of a given radius to be part of the whole sample.

Fig. 1). As a result, Figure 5(c) accounts for the three typical macroscopic stress behaviors presented in dots. In particular, the softening experienced by the dense sample can be linked to the destruction of long force chains. Nevertheless, a slight strain delay is observed between the stress dotted curves and their mean chain length counterparts. This might be related to the three arbitrary thresholds used in the force chain definition recalled in the previous subsection, namely the principal stress threshold, the angle threshold and the minimum length threshold.

Overall, the three graphs in Figure 5 are consistent with the existence of a common critical state with respect to these meso-structures (Zhu et al., 2016) given that the curves corresponding to the different samples collapse on a single curve at the end of the triaxial test.

\subsubsection{Size segregation of chained particles}

Another interesting statistical property of the chained particles with respect to grain detachment is the probability density that a particle of a given radius belongs to a force chain. These probability densities for the three samples considered are shown in Figure 6 Since no noticeable changes can be observed in these probability density functions with respect to the level of axial strain, only the mean density probability functions are plotted together with error bars corresponding to standard deviations. For comparison purposes, the probability density for a grain of a given radius to be part of the different samples is plotted as a dashed line. It should be noted here that the probability density functions are not bounded, but their integral over the whole range of radii is normalized to 1 .

In Figure 6, the probability densities corresponding to chained particles are very different from the reference horizontal prob- 


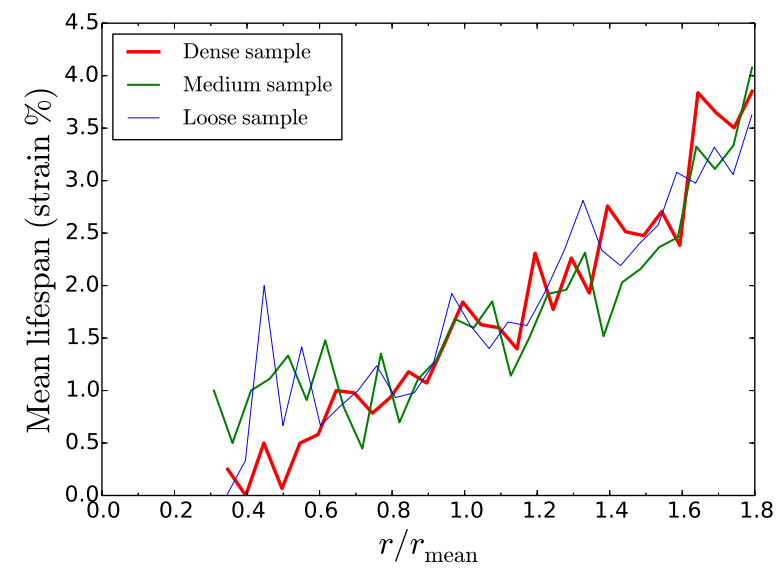

Fig. 7 Averaged lifespans of the chained particles existing before the deviatoric loading in terms of axial strain level $\left|\varepsilon_{z z}\right|$.

ability density for the whole sample imposed by the PSD curve shown in Figure 2. Indeed, force chains are mainly composed of large particles (Cambou et al., 2013, Voivret et al. 2009) and particles smaller than $0.4 r_{\text {mean }}$ are rarely involved in force chains. The dual comment is that the finest particles are the least loaded particles of the samples and therefore the most likely to be detached, provided that fluid forces resulting from an internal flow are large enough. Fine particles are also good candidates for being transported over large distances, which will be estimated in Sect. 4. However, no sharp transition is visible in the probability densities in Figure 6. As a result, no clear radius threshold exists between the loose particles of a granular assembly and the primary fabric responsible for stress transmission, as proposed in many suffusion susceptibility criteria. This result is consistent with the recent findings of To et al. (2015b), proving the existence of an overlapping zone in the PSD curve in which a grain can either belong to the loose or the coarse phase of the resulting granular assembly.

As already mentioned, the probability density functions shown in Figure 6 do not depend on the strain level, which can result either from very stable force chains or from stable statistic rearrangements of chained particles. In order to chose between these two options, the lifespan of a chained particle during the triaxial loading is introduced as the width of the strain interval during which this particle is continuously identified as belonging to force chains. In Figure 7, the average lifespan of the chained particles initially identified in the three samples under the isotropic confining pressure $\sigma_{0}$ is shown with respect to the grains' radii.

As seen in Figure 7, the average lifespan increases with the size of the particles from less than $1 \%$ for $r=r_{\min }$ up to $4 \%$ for $r=r_{\max }$. The force chains containing large particles are therefore less sensitive to an increase in the axial strain and have a longer lifespan than the force chains composed of small particles. However, it can be noted that even for the largest particles, the average lifespan remains limited to a few percent of strain. Overall, it can be concluded that the force chains are constantly rearranging to cope with the deviatoric loading. This dynamic process affects all the particles whatever their radii, but the largest chained particles are more stable than their smaller counterparts. This result is consistent with the recent findings of To et al. (2015b) concerning the evolving populations of loose and primary fabric particles. The comparison between the three graphs in Figure 7 highlights the fact that the initial void ratio does not seem to have a strong influence on this rearrangement process. These results are consistent with the recent findings of Zhu et al. (2016) concerning the force chains' lifespan density probability in the critical state, which mostly follows an exponential decrease with respect to the axial strain.

3.2 Force chain autocorrelation and associated mesoscopic length scales

Even if the statistical properties shown so far are able to describe several salient features of the chained particles, they do not capture the spatial distribution of the force chains. Based on the theoretical work of Kanit et al. (2003), Lantuejoul (1991), Matheron (1967), the spatial distribution of the chained particles can be described using autocorrelation functions.

\subsubsection{Definition of autocorrelation functions}

If $\Omega$ denotes the domain occupied by the chained particles of a given sample of volume $V$, the autocorrelation function $C$ is defined for any vector $\boldsymbol{h}=\left(h_{x}, h_{y}, h_{z}\right)$ as the joint probability that a point $\boldsymbol{x}$ and the translated point $\boldsymbol{x}+\boldsymbol{h}$ simultaneously belong to chained particles (Matheron, 1967):

$C:\left\{\begin{array}{l}\mathbb{R}^{3} \mapsto \mathbb{R} \\ \boldsymbol{h} \mapsto \mathbb{P}\{\boldsymbol{x} \in \Omega \cap \boldsymbol{x}+\boldsymbol{h} \in \Omega \forall \boldsymbol{x} \in V\}\end{array}\right.$

For $\boldsymbol{h}=0$, the autocorrelation $C(0)$ corresponds to the chained particles volume fraction as $C(\boldsymbol{h})=\mathbb{P}\{\boldsymbol{x} \in \Omega\}$, and for $\|\boldsymbol{h}\| \rightarrow \infty, C(\boldsymbol{h})$ converges towards $C(0)^{2}$ because of probability independence $(\mathbb{P}\{\boldsymbol{x} \in \Omega \cap \boldsymbol{x}+\boldsymbol{h} \in \Omega\}=\mathbb{P}\{\boldsymbol{x} \in \Omega\} \times$ $\mathbb{P}\{\boldsymbol{x}+\boldsymbol{h} \in \Omega\}$ ). Thus a normalized autocorrelation function $\tilde{C}$ can be introduced as

$\tilde{C}(\boldsymbol{h})=\frac{C(\boldsymbol{h})-C(0)^{2}}{C(0)-C(0)^{2}}$

When $\|\boldsymbol{h}\|$ varies from 0 to $\infty, \tilde{C}$ varies from 1 to 0 and the rate of decrease characterizes the microstructure autocorrelation distance. A quantitative definition of this autocorre- 
lation distance can be derived from the introduction of an approximate expression as

$$
\tilde{C}_{\text {fit }}(\boldsymbol{h})=\exp \left(-\sqrt{\left(\frac{h_{x}}{L_{x}}\right)^{2}+\left(\frac{h_{y}}{L_{y}}\right)^{2}+\left(\frac{h_{z}}{L_{z}}\right)^{2}}\right),
$$

where $L_{x}, L_{y}$ and $L_{z}$ are three lengths scales that capture the decrease rate of $\tilde{C}$. Even though this expression is empirical, the initial and final theoretical values of $\tilde{C}$ are recovered, the principal directions of geometrical anisotropy imposed by the mechanical loading along the axis $x, y$ and $z$ are respected (Radjai et al., 1998), and as predicted by the theoretical work of Matheron (1967) and reviewed in Corson (1974), the initial tangent of the fit is not equal to zero and close to the specific surface of the samples (not shown here). A refinement of the definition of the three length scales introduced above can be derived from integral range theory (Kanit et al., 2003, Matheron, 1975). It was shown that the variance of a homogenized property $Z$ of a biphasic material computed over a volume $V$ is given by

$D_{Z}^{2}(V)=\phi(1-\phi)(\Delta Z)^{2} \frac{A_{3}}{V}$

where $\Delta Z$ is the property contrast between the two-phases of the material, $\phi$ is the material porosity and $A_{3}$ is the integral range defined as

$A_{3}=\iiint_{\mathbb{R}^{3}} \tilde{C}(\boldsymbol{h}) \mathrm{d} h_{x} \mathrm{~d} h_{y} \mathrm{~d} h_{z}$.

In our case, $Z$ is simply the indicator function associated with force chains ( $Z(x)=1$ if $x \in \Omega, 0$ otherwise) and $\phi$ the porosity computed only by considering chained particles.

Then, the integral range associated with the proposed fit given in equation (5) can be expressed as a function of $L_{x}$, $L_{y}$ and $L_{z}$

$A_{3}^{\mathrm{fit}}=8 \pi L_{x} L_{y} L_{z}$.

Thus a more physical definition of the autocorrelation lengths can be given as

$$
\left\{\begin{array}{l}
L_{x}^{\prime}=2 \pi^{1 / 3} L_{x} \\
L_{y}^{\prime}=2 \pi^{1 / 3} L_{y} \\
L_{z}^{\prime}=2 \pi^{1 / 3} L_{z}
\end{array}\right.
$$

With this definition of the autocorrelation lengths, the integral range related to the chained particles can be seen as a volume of dimensions $\left(L_{x}^{\prime}, L_{y}^{\prime}, L_{z}^{\prime}\right)$. Combined with equation (6), this gives a quantitative definition of the representative elementary volume (REV) associated with force transmission within a given sample as a multiple of the integral range. As a result, this also specifies the REV associated with grain detachment processes, namely a small volume which is structurally entirely typical of the whole material on average and for which the fluctuations due to the imposed boundary conditions can be ignored (Hill, 1963).

It is worth noting here that the integral range can be seen as a virtual unit cell inside which the microstructure is highly correlated. The whole grain assembly may therefore be seen as a collection of unit cells, the shape of which depends on the shape of the autocorrelation $C(\boldsymbol{h})$.

\subsubsection{Autocorrelation between chained particles during triaxial loading}

The autocorrelation functions corresponding to $0,5,10$ and $20 \%$ of deformation are plotted in Figure 8 for the dense sample together with equation (5). Analysis is restricted to the principal directions of loading $\boldsymbol{e}_{x}, \boldsymbol{e}_{y}$ and $\boldsymbol{e}_{z}$. Given that the samples used in this study are surrounded by bounding planes, the boundary particles tend to crystallize in the vicinity of these planes. This effect was highlighted in Reboul et al. (2008) for the local porosity, and the autocorrelation analysis is restricted to the core of the samples to get rid of these boundary effects (effective lengths of 0.8 times the total dimensions of the sample are adopted).

In the initial state, the autocorrelation points for $\boldsymbol{h} \propto \boldsymbol{e}_{x}$ and $\boldsymbol{h} \propto \boldsymbol{e}_{z}$ cannot be distinguished. Accordingly, the autocorrelation lengths $L_{x}^{\prime}$ and $L_{z}^{\prime}$ are very similar in this case, which is consistent with the fact that under the isotropic initial confining pressure the force chains tend to follow an isotropic distribution, as illustrated in Figure 4 In the final state, the autocorrelation function converges toward 0 much more slowly in the $z$ direction than in the $x$ direction. This is consistent with the fact that the force chains tend to align along the vertical principal direction of loading as seen in Figure 4. Thus the autocorrelation lengths $L_{x}^{\prime}$ and $L_{z}^{\prime}$ are able to account for this anisotropic evolution of the microstructure. However, the proposed fit systematically overestimates the horizontal autocorrelation and cannot account for the negative values obtained for the horizontal autocorrelation function for $\left|\varepsilon_{z z}\right| \geq 5 \%$. This latter anti-correlation feature should be related to an exclusion zone around force chains in the horizontal plane located at about $3 r_{\text {mean }}$.

More comprehensively, the three autocorrelation lengths are plotted against the axial strain for the three samples considered in Figure 9 (a). A similar analysis is performed for the non-chained particles in Figure 9(b).

In Figure 9 (a), as the horizontal autocorrelation lengths remains more or less constant for all the samples around $3 r_{\text {mean }}$ for the chained particles, the vertical autocorrelation length increases significantly between the initial and the final state. For the loose and the medium samples, the increase is monotonous whereas the vertical autocorrelation length for the dense sample reaches a peak value around $4.5 r_{\text {mean }}$ for the chained particles. For $\left|\varepsilon_{z z}\right|>15 \%$, a critical state is reached as $L_{z}^{\prime}$ stabilizes for all the samples around $4 r_{\text {mean }}$ for the chained 

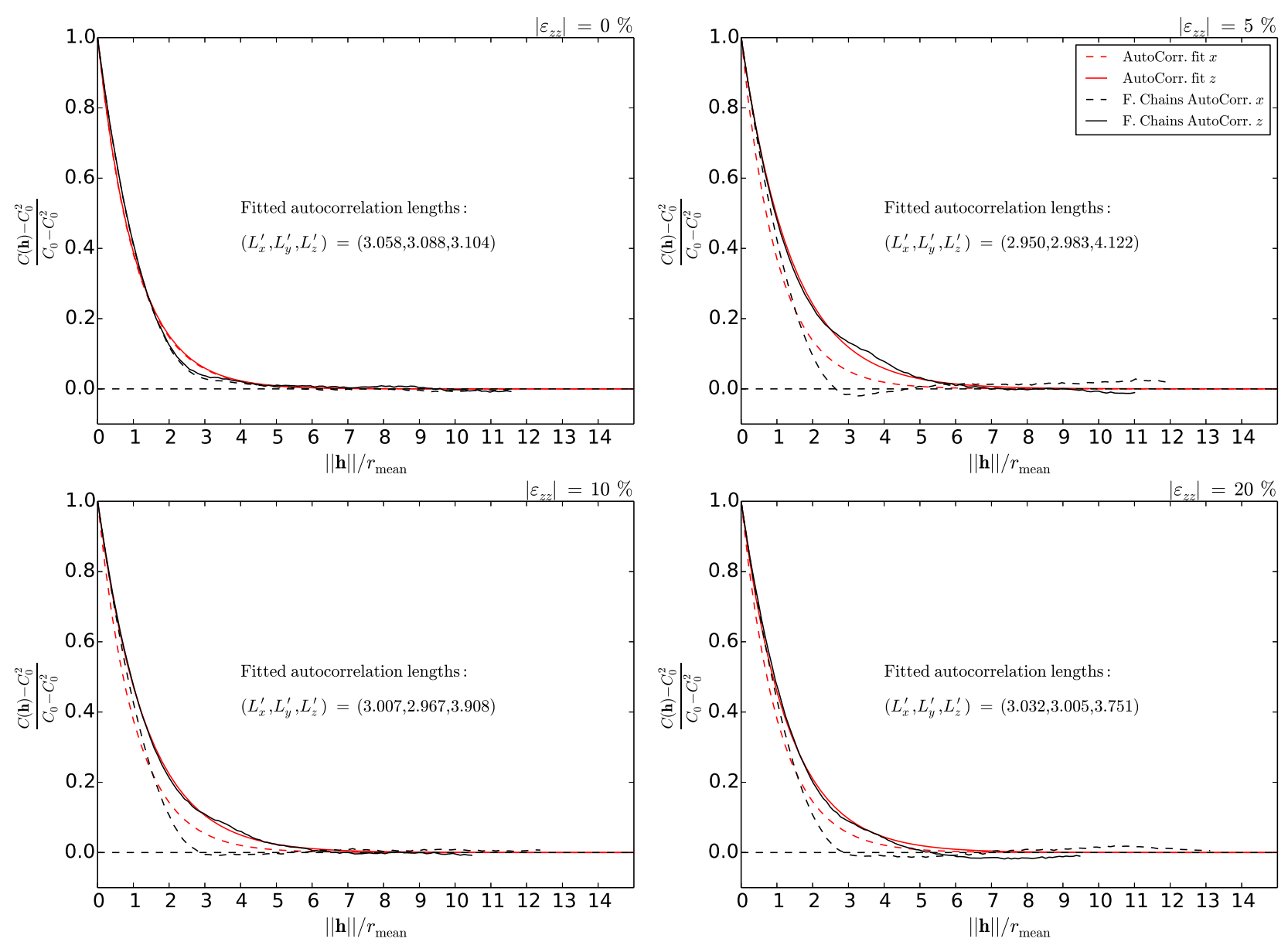

Fig. 8 Rescaled autocorrelation functions computed for the dense sample at different strain levels $(|\varepsilon| \in\{0,0.05,0.1,0.2\})$. The plot of $\tilde{C}$ is restricted to $\boldsymbol{h} \propto \boldsymbol{e}_{x}$ and $\boldsymbol{h} \propto \boldsymbol{e}_{z}$. The corresponding fitted autocorrelation functions $\tilde{C}_{\text {fit }}$ are shown and the estimated autocorrelation lengths $L_{i}^{\prime}$ are given.

particles. At this stage, the spatial distribution of chained particles can be characterized by a small volume, the size of which is

$\left(L_{x}^{\prime}, L_{y}^{\prime}, L_{z}^{\prime}\right)=\left(3 r_{\text {mean }}, 3 r_{\text {mean }}, 4 r_{\text {mean }}\right)$

With reference to equation (6), this gives the order of magnitude of the REV associated with the stress distribution within the different samples. For the sake of illustration, the volume fraction occupied by chained particles for the dense sample in the isotropic compression state is $30 \%$. An estimation of this volume fraction with a variance of $10^{-4}$ would require a volume $V$ equal to roughly 2000 times bigger than $A_{3}$ corresponding to a REV of dimensions $\left(38 r_{\text {mean }}, 38 r_{\text {mean }}, 50 r_{\text {mean }}\right)$ which is close to the total size of our sample.

In Figure 9 (b) the evolution of the autocorrelation lengths computed for the non-chained particles is qualitatively similar to the one corresponding to chained particles. This argues in favor of the fact that the spatial distribution of loose particles can be effectively described by the spatial distribution of chained particles. However, the autocorrelation lengths $L_{i}^{\prime}$ are slightly smaller when considering the non-chained particles instead of the chained ones which could be related to the slight misfit observed in Figure 8 with respect to the horizontal autocorrelation for $\left|\varepsilon_{z z}\right|>0 \%$. When looking at the transient evolution of $L_{z}^{\prime}$ with respect to $\varepsilon_{z z}$, it can be noted that the dense sample is characterized by an initial increase in the vertical autocorrelation followed by a decrease toward the critical state. This can be closely related to the macroscopic response observed in Figure 3 . Indeed the increase in the vertical autocorrelation corresponds to a lengthening of the force chains along the vertical direction which accounts for the initial hardening of the dense sample. The following decrease in the vertical autocorrelation accounts for the destruction of force chains and thus to the stress softening observed for the dense sample. For the medium and 


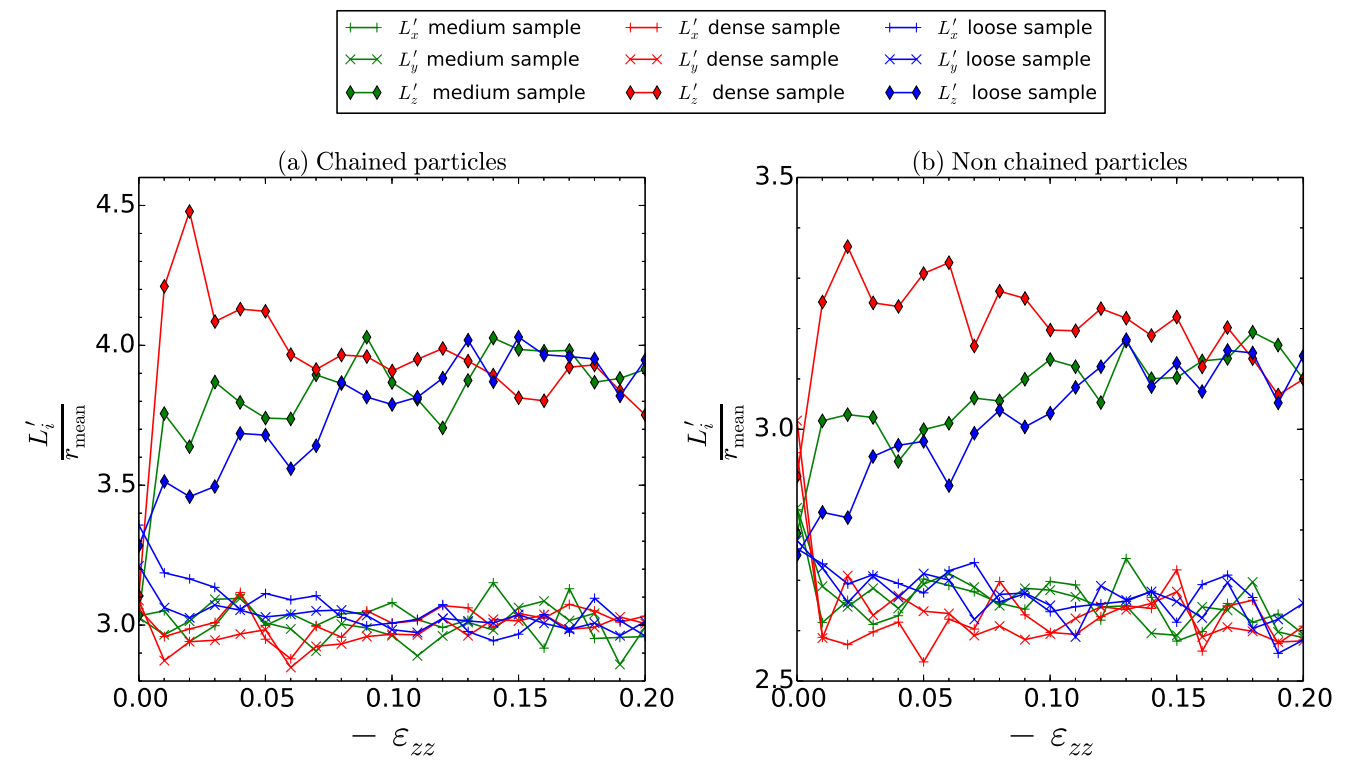

Fig. 9 Strain evolution of the three autocorrelation lengths $L_{i}^{\prime}(i \in\{x, y, z\})$ during the triaxial test. Analysis is restricted either to particles belonging to force chains (a) or to particles not belonging to force chains (b).

the loose samples, $L_{z}^{\prime}$ increases more or less continuously until reaching the critical state. This is consistent with the fact that these two samples do not experience any softening. In the end of the triaxial test, the three samples exhibit a common mesostructure associated with force chains whose size is characterized by equation 10 . This provides a quantitative definition of the force chain pattern existing in the critical state as pointed out by Zhu et al. (2016).

\section{Mesoscale investigation with respect to particle transport}

The microscale analysis of the pore network is critical to understanding the transport of free particles through a granular matrix after being detached from the granular skeleton. If we envision for instance a possible homogenization approach, identifying the typical length scale associated with the transport of particles and comparing it to the scale monitoring the grain detachment process studied in the previous section stands as a critical issue.

\subsection{Pore network definition}

In the wake of the previous work of Reboul et al. (2008) and Vincens et al. (2015) for the transport of spherical cohesionless particles, the pore space can be reduced to the definition of a pore network. A regular triangulation of a spheres assembly (Edelsbrunner and Shah, 1996) is built from YADE software. This particular type of triangulation has the important property that all the edges of its dual tessellation be- long to the pore space (Chareyre et al. 2012, Vincens et al. 2015).

This tessellation is the key ingredient in defining a pore network composed of pores (the nodes of the graph) and constrictions (the edges of the graph). The pores are defined following the level 0 analysis introduced in Reboul et al. (2008). Their positions are defined at the center of each tetrahedron of the regular triangulation in terms of power distances and their radii as the radius of the largest interior sphere in the associated tetrahedra. The constrictions are modeled as cylinders joining two adjacent pores (Reboul et al. 2008) and their radii are defined as the radius of the largest interior circle on the common face of the two tetrahedra defining the constriction. The definition of the pore and constriction radii is shown in Figure 10 (a) and (b). For the sake of illustration, three-dimensional visualizations of the pore network associated with two simple grain assemblies are shown in Figures 10 (c) and (d). It should also be noticed that the considered triangulation is built while considering all the particles, including the potentially migrating particles identified thanks to the force chains analysis. This choice is motivated by the idea that only a few non-chained particles will actually be detached under the action of a fluid, leading to few changes in the initial pore network.

The bounding planes surrounding the samples locally induce a very different microstructure from that observed in the core of the samples. Indeed, close to the boundary, a significant number of tetrahedra are found to be flat, which results in the estimation of very huge pore and constrictions radii. Consequently, the pore network is only defined on a centered subvolume fraction of $0.8^{3}$. A visualization of the 


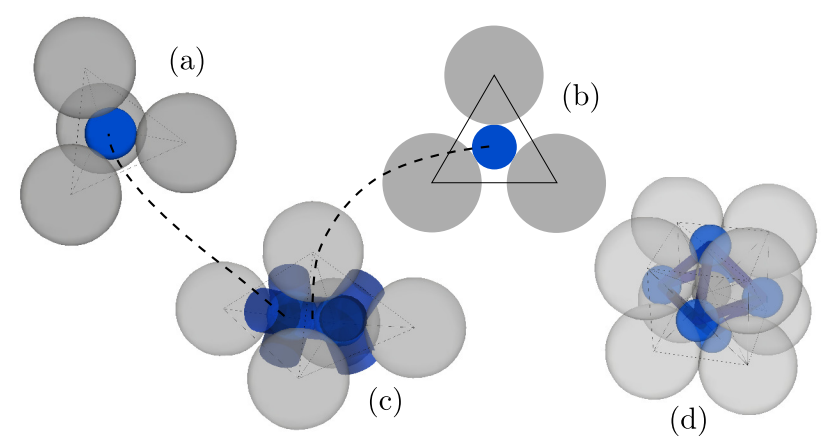

Fig. 10 Visualization of a tetrahedron with its inscribed pore sphere (a), a tetrahedron face with its inscribed constriction circle (b), a simple assembly of four spheres with the associated pore network (c), and an assembly of nine sphere with the associated pore network (d). In these figures, the spherical particles are represented in grey, the pore by blue spheres and the constrictions between two pores by blue cylinders. The tetrahedra resulting from the regular Delaunay triangulation are represented with black lines joining the different particles centers.

pore network for the dense sample at the beginning and the end of the triaxial test is given in Figure 11.

In this figure the constrictions are represented by grey-scaled cylinders linking two pore centers. The lighter the cylinders, the greater the constrictions. The whole pore network is visible in snapshots $\left(a_{i}\right)$ and $\left(b_{i}\right)$ in Figure 11 The network is very dense and enables many possible paths connecting the different pores of the sample. To show only sufficiently large constrictions that allow particle transport, a radius threshold is applied. The two snapshots $\left(\mathrm{a}_{\mathrm{ii}}\right)$ and $\left(\mathrm{b}_{\mathrm{ii}}\right)$ correspond to a radius threshold $r=0.3 r_{\text {mean }}$, and the two snapshots $\left(\mathrm{a}_{\mathrm{iii}}\right)$ and $\left(\mathrm{b}_{\mathrm{iii}}\right)$ correspond to a radius threshold $r=0.5 r_{\text {mean }}$. In both cases, sufficiently small particles exist in the sample to be transported through the represented constrictions (see Figure 12). Although many constrictions larger than $0.3 r_{\text {mean }}$ are still connected together, constrictions larger than $0.5 r_{\text {mean }}$ are isolated from one another. These features give an idea of the expected transport distances in the pore space for particles of different radii. The comparison between Figure 11 (a) $\left(\left|\varepsilon_{z z}\right|=0 \%\right)$ and Figure 11 (b) $\left(\left|\varepsilon_{z z}\right|=20 \%\right)$ highlights the fact that the number of large constrictions increases between the beginning and the end of the triaxial test for the dense sample, which is consistent with its dilative behavior observed in Figure 3.

\subsection{Statistical identification of potentially transportable particles}

From the pore network definition, the probability densities corresponding to pore and constriction radii are computed and plotted in Figure 12 for the three samples considered at four strain levels. To identify whether some particles of the sample could be transported within the pore space, the density probabilities that a grain and a non-chained grain would be of a given radius (see Figure 6) are also reported in Figure 12 .

For every sample in Figure 12, the pore and the constriction probability density function follows single-mode distributions around $r=0.25 r_{\text {mean }}$ and $r=0.5 r_{\text {mean }}$, respectively. Since the constriction mode value $\left(r=0.2 r_{\text {mean }}\right)$ is significantly smaller than the pore value $\left(r=0.5 r_{\text {mean }}\right)$, the transport of particles is governed mainly by the constriction sizes and not by the pore sizes. For the three samples, the comparison between the constriction probability density and that corresponding to the grains highlights that a large number of grains are small enough to be transported through the pore network. An even larger fraction of the non-chained grains is concerned.

While observing how the strain of the pore and constriction probability densities evolved, the contractive/dilative behavior observed in Figure 3 is recovered. For the dense sample, the probability densities are shifted toward larger radius values, which is related to an overall increase of the pore space volume. This observation is consistent with the dilative behavior observed in Figure 3 . On the contrary, for the medium and the loose samples, the probability densities are shifted toward slightly smaller radius values, which accounts for a decrease in the pore space volume.

\subsection{Mean travel distance functions and associated mesoscopic length scales}

Even though many particles are identified in Figure 12 as potentially detachable by analyzing the statistical properties of the pore network, determining the distance that a particle of a given radius can cover requires knowing the spatial distribution of pore and constriction radii. This distribution is accessible from the graph description of the pore space (see Figure 11. As constriction radii are smaller than their pore counterparts (Figure 12), only the constriction radii are considered while assessing the possibility for a grain to move from one pore to another. This is the reason why pores are not represented in Figure 11 .

\subsubsection{Pore-to-pore transport criteria}

In a granular material subjected to an internal flow, particle transport is not isotropic and is governed by the direction and intensity of the flow through the pore space of the media. Therefore, the transport properties of the pore networks defined for the three samples are considered with a horizontal fluid flow imposed by a small pressure gradient of $10 \mathrm{~Pa} \ll \sigma_{0}$ between the sample boundaries in the $x$ direction. It should be noted that in hydraulic structures the flow direction is most often perpendicular to the principal direction of loading and therefore is mostly horizontal. This 
Author-produced version of the article published in Granular MAtter, 2017, 19, 22.

The original publication is available at https://link.springer.com/article/10.1007/s10035-017-0706-9

DOI: $10.1007 /$ s10035-017-0706-9
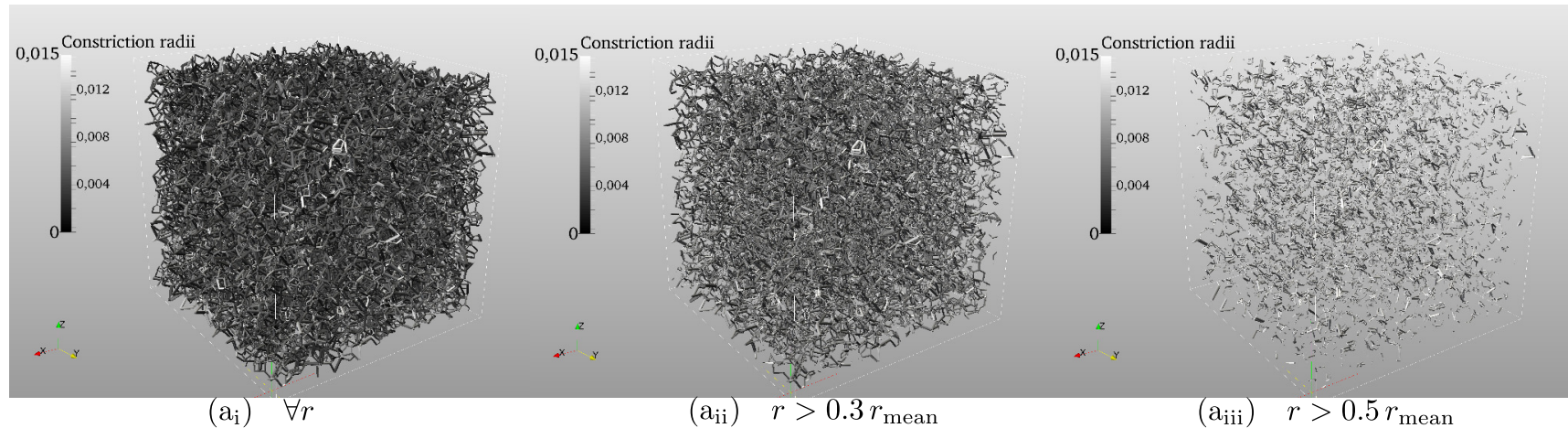

(a) $\left|\varepsilon_{z z}\right|=0 \%$

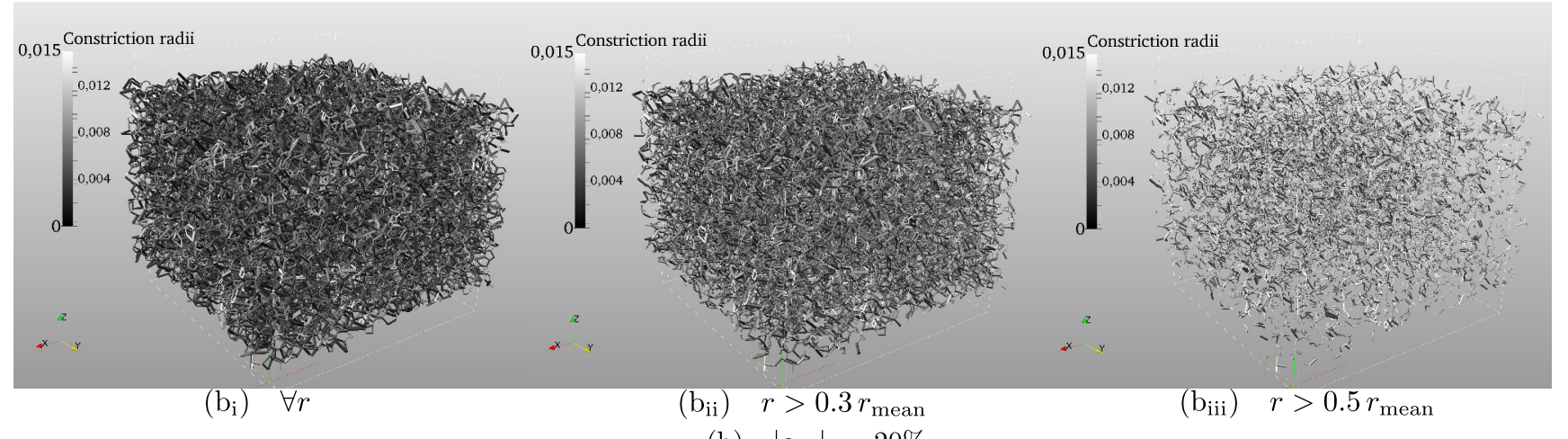

(b) $\left|\varepsilon_{z z}\right|=20 \%$

Fig. 11 Three dimensional visualization of the dense sample pore network under the initial isotropic loading (a) and after $20 \%$ of deformation (b). Only constrictions are represented thanks to cylinders. The lighter the constrictions, the larger their radii. On the left, all constrictions are visible. In the middle, a radius threshold of $0.3 r_{\text {mean }}$ is applied. On the right, a radius threshold of $0.5 r_{\text {mean }}$ is applied.

fluid/grain problem is addressed using the DEM-PFV model implemented in YADE (Chareyre et al. 2012).

From these simulations a pore pressure map is defined and used to study the transport of particles in the pore network. Based on the Hagen-Poiseuille flow velocity profile for a circular tube and the expression of the drag force of a uniform flow acting on a sphere for low Reynolds numbers (Fig. 13), it can be assumed that the drag force acting on a particle of radius $r$ in a constriction of radius $R$ and subjected to a pressure gradient $\boldsymbol{\nabla} \boldsymbol{p}$ scales as

$$
F \sim r \nabla p R^{2}
$$

From this simple scaling law, a criterion for particle transport is established. Given a pore $i$ connected to neighboring pores $\left\{j_{1}, j_{2}, j_{3}, j_{4}\right\}$, a transported particle of radius $r$ in the pore $i$ will move to a neighboring pore $k \in\left\{j_{1}, j_{2}, j_{3}, j_{4}\right\}$ if and only if

$$
\left\{\begin{array}{l}
R_{i k}>r \\
\left\|\nabla \boldsymbol{p}_{i j}\right\| R_{i j}^{2} \leq\left\|\boldsymbol{\nabla} \boldsymbol{p}_{i k}\right\| R_{i k}^{2} \quad \forall j \in\left\{j_{1}, j_{2}, j_{3}, j_{4}\right\}
\end{array} .\right.
$$

In other words, the particle propagates to the next accessible pore in the direction of maximum drag force.

\subsubsection{Definition of a transport susceptibility criterion}

To estimate the average distance a particle of radius $r$ can travel in the pore space, a propagation path $\mathscr{P}\left(n_{0}, r\right)$ is defined for every node $n_{0}$ of the pore network by repeated application of the propagation criterion introduced in equation (12). For any node $n_{0}$, the farthest node $n_{k}$ that the particle considered can reach is thus computed. From the list of the visited nodes $\left\{n_{0}, \ldots n_{k}\right\}$, the true travel distance $D\left(n_{0}, r\right)$ and the tortuosity $T\left(n_{0}, r\right)$ corresponding to the path $\mathscr{P}\left(n_{0}, r\right)$ are then computed as

$$
\left\{\begin{array}{l}
D\left(n_{0}, r\right)=\sum_{i=0}^{k-1}\left\|\boldsymbol{x}_{i+1}-\boldsymbol{x}_{i}\right\| \\
T\left(n_{0}, r\right)=\frac{D\left(n_{0}, r\right)}{\left\|\boldsymbol{x}_{k}-\boldsymbol{x}_{0}\right\|}
\end{array}\right.
$$

where $\boldsymbol{x}_{i}$ stands for the vector position of node $n_{i}$. Figure 14 provides examples of propagation paths computed for the dense sample under the initial isotropic confining pressure $\sigma_{0}$ and for a radius threshold $r=0$. On average, all the visible paths are aligned with the horizontal direction $x$ corresponding to the imposed flow pressure gradient. As the radius threshold is chosen as $r=0$, all the paths end on the sample boundaries, which is consistent with the fact that the pore space in $3 \mathrm{D}$ for a sphere assembly is a connected space. 

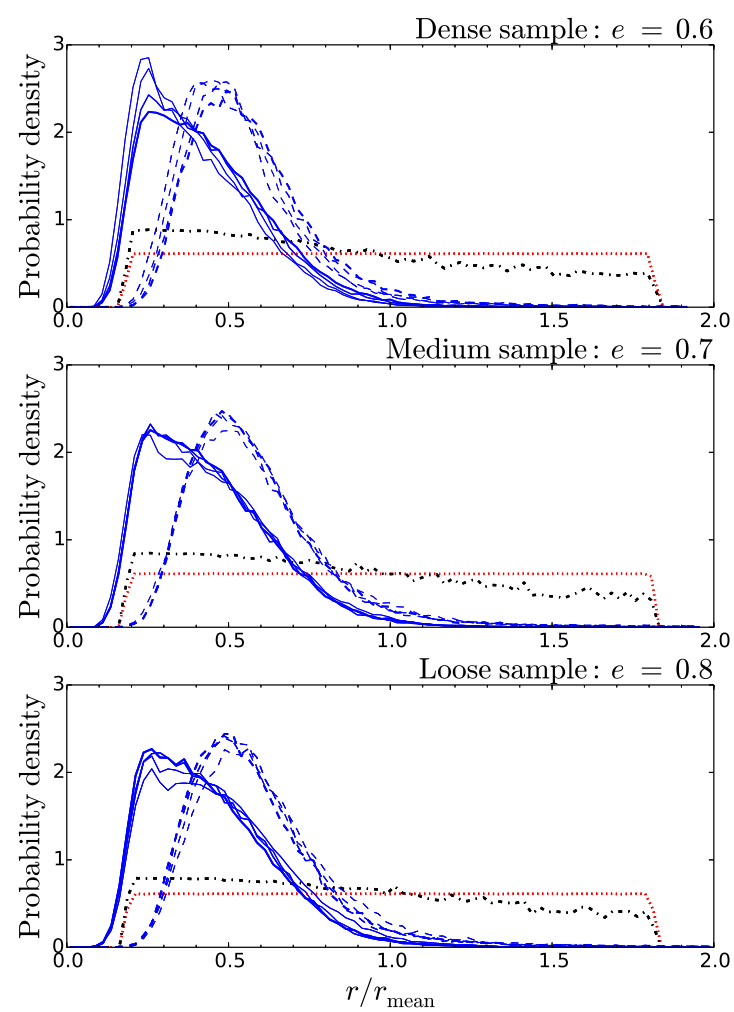

Fig. 12 Probability density for a pore (dashed blue), a constriction (solid blue), a grain (dotted red) or a non-chained grain (dash-dotted black) to be of a given radius. The three graphs correspond to the three samples used in this study. The line thickness corresponds to the strain level $\left|\varepsilon_{z z}\right| \in\{0,0.05,0.1,0.2\}$ during the triaxial test. The thicker the line, the larger the strain.

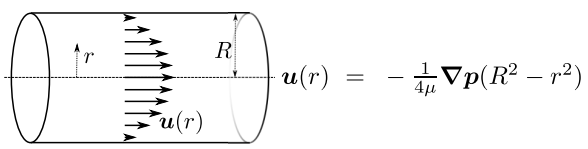

(a)

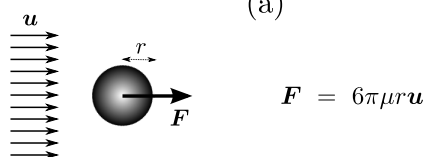

(b)

Fig. 13 Hagen-Poiseuille flow velocity profile in a circular tube (a) and Stokes drag force (b). In both cases viscous flow conditions are assumed.

The mean travel distance $\bar{D}(r)$ and the mean tortuosity $\bar{T}(r)$ are then deduced from statistical averaging of the above quantities over all the nodes of the pore network. In Figure 15 the mean travel distance is plotted together with the mean tortuosity for different radius values at four strain levels.

For the smallest radius values, the mean travel distance reaches a plateau, which is related to a percolation phenomenon. Indeed, the transport path can end up on the boundary of the sample for some nodes, as seen in Figure 14 In this case, the end of the propagation path does not coincide with the ac-

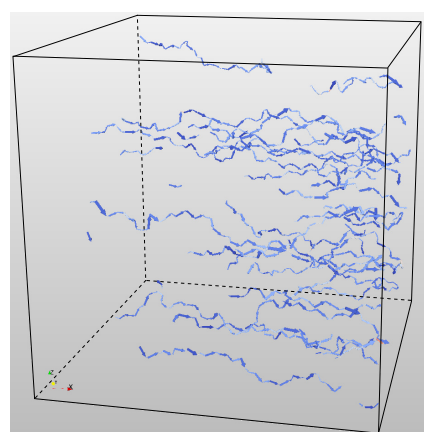

Fig. 14 Visualization of 60 propagation paths for the dense sample under the initial isotropic confining pressure $\sigma_{0}$. The paths are chosen randomly for a radius threshold $r=0$. The arrows indicate the propagation direction. The more intense the blue color, the larger the constriction radius. All paths end on the boundaries of the sample materialised by an empty cube.

tual farthest point the particle would reach in a non-bounded sample. Consequently, such events are dismissed in the averaging process. In Figure 15 a percolation phenomenon is observed for $r$ smaller than $0.2 r_{\text {mean }}$ for all the samples. Conversely, no transport is possible for particles larger than $0.5 r_{\text {mean }}$ for all the samples. This observation is consistent with the findings of Reboul et al. (2008) highlighting the fact that the connectivity of the pores larger than the radius mode value of the probability density function on Figure 12 is too small to allow the transport of such large particles. The grey zone on the graphs corresponds to the range of radius values for the grains constituting the samples. If the largest grains cannot be transported, the smallest ones can be transported over long distances up to $25 r_{\text {mean }}$. However, no percolation exists since there is no overlapping between the initial percolation plateau and grey zones on the graphs. Because of its dilative behavior (see Figure 3), transport appears to be greater in the dense sample after a few percent of vertical strain. This is not the case in the initial state (thin curves) where the mean travel distances increase as the sample densities decrease. For the loose and medium samples that exhibit a contractive behavior, the mean travel distance is reduced for the largest particles, but the mean travel distance of the smallest particles is slightly increased.

The dashed curves in Figure 15 represent the evolution of the tortuosity of the transport paths. No noticeable difference is visible between the three samples and the mean tortuosity decreases from 1.5 to 1 as the particle size increases following the mean change in travel distance. The limit value of 1 corresponds to the case where particles are so large that they are trapped in one or two pores. As the mean travel distance increases, the mean tortuosity increases and stabilizes around a finite value. This should be related to the fact that the propagation is driven by the flow direction, the mean direction of which is parallel to the $x$ direction with limited fluctuations along the cross directions. 

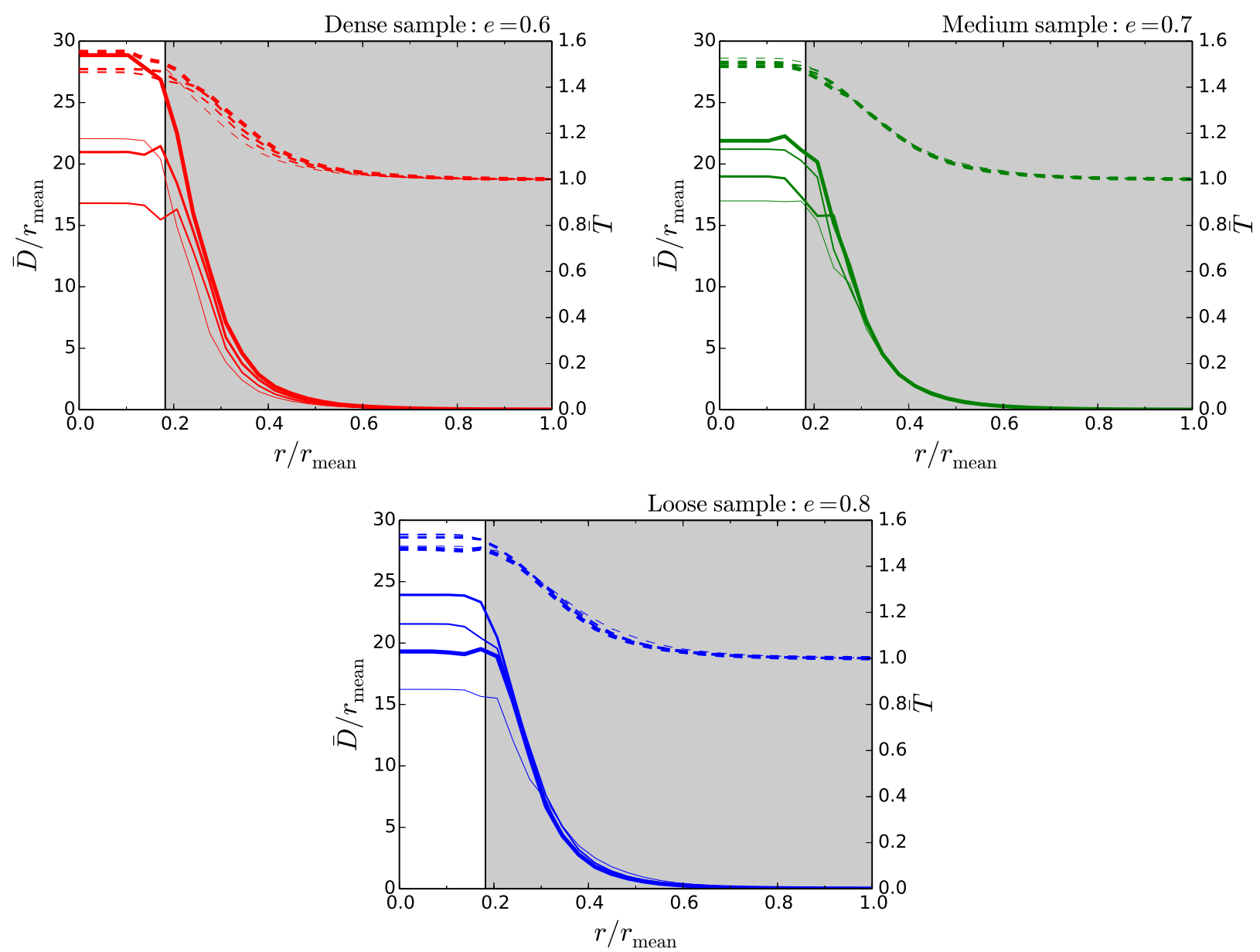

Fig. 15 Mean travel distance (solid lines) and mean tortuosity (dashed lines) for different radius thresholds. The three graphs correspond to the three samples used in this study and the line thickness corresponds to the strain level $\left|\varepsilon_{z z}\right| \in\{0,0.05,0.1,0.2\}$ during the triaxial test. The thicker the line, the bigger the strain. The grey domain in the background corresponds to the accessible radius values for the grains composing the samples.

\section{Conclusions}

Specific micromechanical tools are developed in this paper to investigate the susceptibility of a polydisperse assembly of spherical particles to grain detachment and grain transport. Basically, the granular assembly can be considered as a two-phase medium. A partition of its solid fraction between chained particles and non-chained particles makes it possible to analyze the grain detachment process. Similarly, the void fraction can be split into two parts corresponding to an accessible pore network and a non-accessible pore network. This dual partition is a fundamental ingredient to describe the grain transport process.

Based on DEM simulations, the microstructure's evolution is recorded during a triaxial loading and the relevance of the proposed micromechanical tools in correctly predicting the observed macroscopic behavior is investigated. In particular, the ability of the chained particles' autocorrelation lengths to recover the hardening and softening behaviors of the dense sample confirms the relevance of this approach to describe the typical length scales associated with stress transmission. Likewise, the mean travel distance functions are shown to address the dilative and contractive behaviors of the specimen considered.

By simultaneously considering the size distributions of the non-chained particles and the constrictions of the pore network, the ability of a fluid flow to modify the microstructure of the samples is analyzed with respect to the fraction of both potentially detachable and transportable particles.

By carefully considering the spatial distribution of particles participating in stress transmission and the spatial distribution of constrictions enabling particle transport within the pore space, two mesoscales are introduced to study the grain detachment and the grain transport processes from a micromechanical point of view. It was shown that the typical length scale associated with grain transport can be up to ten times larger than that associated with grain detachment. As a result, a scale separation should exist between these two pro- 
cesses for the smallest particles and for the used particle size distribution. This implies that grain detachment and grain transport can be studied independently from one another. Macroscopically, if this separation of scales holds, suffusion may be described as the superposition of a detachment and a transport processes.

Eventually, full 3D modeling of the suffusion process on a sufficiently large sample with respect to the two mesoscales is accessible and can be used to confirm this scale separation. This would pave the way for the definition of a homogenized law for suffusion thanks to the use of representative elementary volumes sufficiently big with respect to the grain detachment and transport mechanisms.

Conflict of interest The authors declare that there is no conflict of interests regarding the publication of this article. Publication has been approved by all authors. None of the material presented in the paper is submitted or published elsewhere.

\section{References}

Šmilauer et al., V.: Yade Documentation 2nd ed. The Yade Project (2015). DOI 10.5281/zenodo.34073. Http://yadedem.org/doc/

Bonelli, S.: Erosion of geomaterials. John Wiley \& Sons (2012)

Cambou, B., Jean, M., Radjai, F.: Micromechanics of granular materials. John Wiley \& Sons (2013)

Chareyre, B., Cortis, A., Catalano, E., Barthélemy, E.: Porescale modeling of viscous flow and induced forces in dense sphere packings. Transport in porous media 94(2), 595-615 (2012). DOI 10.1007/s11242-011-9915-6

Corson, P.B.: Correlation functions for predicting properties of heterogeneous materials. ii. empirical construction of spatial correlation functions for two-phase solids. Journal of applied Physics 45(7), 3165-3170 (1974). DOI 10. 1063/1.1663742

Cundall, P.A., Strack, O.D.: A discrete numerical model for granular assemblies. Geotechnique 29(1), 47-65 (1979). DOI 10.1680/geot.1979.29.1.47

Edelsbrunner, H., Shah, N.R.: Incremental topological flipping works for regular triangulations. Algorithmica 15(3), 223-241 (1996)

Fonseca, J., Sim, W., Shire, T., O'Sullivan, C.: Microstructural analysis of sands with varying degrees of internal stability. Géotechnique 64(5), 405-411 (2014). DOI 10.1680/geot.13.T.014

Hadda, N.: Aspects micromécaniques de la rupture dans les milieux granulaires. Ph.D. thesis, Ecole Doctorale Ingénierie - Matériaux Mécanique Environnement Energétique Procédés Production (I-MEP ${ }^{2}$ ) (2006)
Hill, R.: Elastic properties of reinforced solids: some theoretical principles. Journal of the Mechanics and Physics of Solids 11(5), 357-372 (1963)

Kanit, T., Forest, S., Galliet, I., Mounoury, V., Jeulin, D.: Determination of the size of the representative volume element for random composites: statistical and numerical approach. International Journal of solids and structures 40(13), 3647-3679 (2003). DOI 10.1016/ S0020-7683(03)00143-4

Kenney, T., Lau, D.: Internal stability of granular filters. Canadian Geotechnical Journal 22(2), 215-225 (1985). DOI 10.1139/t85-029

Kézdi, Á.: Soil physics: selected topics, vol. 25. Elsevier (2013)

Langroudi, M.F., Soroush, A., Shourijeh, P.T.: A comparison of micromechanical assessments with internal stability/instability criteria for soils. Powder Technology 276, 66-79 (2015). DOI 10.1016/j.powtec.2015.02.014

Lantuejoul, C.: Ergodicity and integral range. Journal of Microscopy 161(3), 387-403 (1991). DOI 10.1111/j. 1365-2818.1991.tb03099.x

Li, W., Vincens, E., Reboul, N., Chareyre, B.: Constrictions and filtration of fine particles in numerical granular filters: Influence of the fabric within the material. In: Scour and Erosion: Proceedings of the 7th International Conference on Scour and Erosion, Perth, Australia, 2-4 December 2014, p. 241. CRC Press (2014)

Matheron, G.: Eléments pour une théorie des milieux poreux. Masson (1967)

Matheron, G.: Random sets and integral geometry. John Wiley \& Sons (1975)

OSullivan, C., Bluthé, J., Sejpar, K., Shire, T., Cheung, L.: Contact based void partitioning to assess filtration properties in dem simulations. Computers and Geotechnics 64, 120-131 (2015). DOI 10.1016/j.compgeo.2014.11.003

Peters, J.F., Muthuswamy, M., Wibowo, J., Tordesillas, A.: Characterization of force chains in granular material. Physical review E 72(4), 041,307 (2005). DOI 10.1103/ PhysRevE.72.041307

Radjai, F., Wolf, D.E., Jean, M., Moreau, J.J.: Bimodal character of stress transmission in granular packings. Physical review letters 80(1), 61 (1998)

Reboul, N., Vincens, E., Cambou, B.: A statistical analysis of void size distribution in a simulated narrowly graded packing of spheres. Granular Matter 10(6), 457-468 (2008). DOI 10.1007/s10035-008-0111-5

Schofield, A., Wroth, C.: Critical state soil mechanics. McGrow-Hill, London (1968)

Scholtès, L., Hicher, P.Y., Sibille, L.: Multiscale approaches to describe mechanical responses induced by particle removal in granular materials. Comptes Rendus Mécanique 338(10), 627-638 (2010). DOI 10.1016/j.crme.2010.10. 003 
Shire, T., O'Sullivan, C.: Micromechanical assessment of an internal stability criterion. Acta Geotechnica 8(1), 81-90 (2013). DOI 10.1007/s11440-012-0176-5

Sibille, L., Marot, D., Sail, Y.: A description of internal erosion by suffusion and induced settlements on cohesionless granular matter. Acta Geotechnica 10(6), 735-748 (2015). DOI 10.1007/s11440-015-0388-6

Sjah, J., Vincens, E.: Determination of the constriction size distribution of granular filters by filtration tests. International Journal for Numerical and Analytical Methods in Geomechanics 37(10), 1231-1246 (2013)

Terzaghi, K.: 45th james forrest lecture, 1939. soil mechanics-a new chapter in engineering science. Journal of the ICE 12(7), 106-142 (1939)

Terzaghi, K., Peck, R.B., Mesri, G.: Soil mechanics in engineering practice. John Wiley \& Sons (1996)

To, H., Scheuermann, A.: Separation of grain size distribution for application of self-filtration criteria in suffusion assessment. In: Scour and Erosion: Proceedings of the 7th International Conference on Scour and Erosion, Perth, Australia, 2-4 December 2014, p. 121. CRC Press (2014)

To, H.D., Scheuermann, A., Galindo-Torres, S.A.: Probability of transportation of loose particles in suffusion assessment by self-filtration criteria. Journal of Geotechnical and Geoenvironmental Engineering p. 04015078 (2015a). DOI 10.1061/(ASCE)GT.1943-5606.0001403

To, H.D., Torres, S.A.G., Scheuermann, A.: Primary fabric fraction analysis of granular soils. Acta Geotechnica 10(3), 375-387 (2015b). DOI 10.1007/ s11440-014-0353-9

Tordesillas, A., Walker, D.M., Lin, Q.: Force cycles and force chains. Physical Review E 81(1), 011,302 (2010)

Vincens, E., Witt, K.J., Homberg, U.: Approaches to determine the constriction size distribution for understanding filtration phenomena in granular materials. Acta Geotechnica 10(3), 291-303 (2015). DOI 10.1007/ s11440-014-0308-1

Voivret, C., Radjai, F., Delenne, J.Y., El Youssoufi, M.S.: Multiscale force networks in highly polydisperse granular media. Physical review letters 102(17), 178,001 (2009). DOI 10.1103/PhysRevLett.102.178001

Wang, X., Li, J.: On the degradation of granular materials due to internal erosion. Acta Mechanica Sinica 31(5), 685-697 (2015). DOI 10.1007/s10409-015-0466-x

Zhu, H., Nguyen, H.N., Nicot, F., Darve, F.: On a common critical state in localized and diffuse failure modes. Journal of the Mechanics and Physics of Solids (2016). DOI 10.1016/j.jmps.2016.05.026 